\title{
Preliminary study of the pilot's workload during emergency procedures in helicopters air operations
}

\author{
Flávio G. V. Bezerra ${ }^{\mathrm{a}^{*}}$ and Selma L. O. Ribeiro ${ }^{\mathrm{b}, \mathrm{c}}$ \\ ${ }^{\mathrm{a} b}$ Aeronautical Engineering Division (IEA.) Technological Institute of Aeronautics (ITA). Professional Master \\ Course in Aviation Safety and Continued Airworthiness. \\ ${ }^{a}$ Secretary of Public Security of the State of Mato Grosso, Integrated Air Operations Center - CIOPAer, \\ Marechal Rondon International Airport, Hangar State, Av. Governador Ponce de Arruda, S/N $N^{0}$, Jardim \\ Aeroporto, Varzea Grande-MT, Brasil, ZC.78.110-971 \\ ${ }^{\mathrm{b}}$ National Institute for the Spatial and Aeronautical Development - IDEA, Av. Franklin Roosevelt, 194, gr. 507, \\ Centro Rio de Janeiro-RJ, Brasil, Z.C.20.021-120 \\ ${ }^{\mathrm{c}}$ Estácio de Sá University
}

\begin{abstract}
Military air operations and law enforcement operations in helicopters are examples of activities that require high performance of the operator. This article aimed at presenting a preliminary analysis of data obtained in the initial study in order to validate the instruments and the research protocol that focuses on the analysis of the workload imposed on helicopter pilots in emergency situations. The research was conducted in an environment of real flight training and used the NASA-TLX Scale to assess the workload and an interview guide to obtain reports on the main tasks performed. Preliminary data obtained is related to the participation of 10 (ten) volunteer pilots with experience in different types of helicopters. Four scenarios involving helicopter emergency procedures of HB-350 "Squirrel" were outlined. For this article, the analysis used only the data regarding the Physical and Mental Demands of the NASA-TLX Scale. Preliminarily, the results indicate that the "time pressure" as a factor contributing to increase mental requirement in emergency situations in flight, and that this increase was reflected in the request of mental processes such as: identification of the breakdown, attention and monitoring parameters. Future steps include extending the sample and adding physiological tools to better understand the effects of these types of emergencies on pilot performance and flight safety.
\end{abstract}

Keywords: workload, NASA-TLX scale, helicopter emergency, subjective measures, mental demand.

\section{Introduction}

Recently the Brazilian society was appalled with the wide press coverage of a police operation, in the northern part of the city of Rio de Janeiro, in which criminals have responded to a police intervention and shot the Phoenix 03 helicopter, from the Military Police of Rio de Janeiro State. As a result, the pilot was forced to make an emergency landing, where three of the six policemen were killed and the aircraft was totally consumed by fire.
The case aroused the curiosity about the limitations of the pilot to operate a helicopter in degraded operating conditions, having to carry out emergency procedures by the manual, even at high risk of death. Mainly because the military and law enforcement missions, by the very nature of the activity, impose a greater demand for physical procedures and mental processes by the pilot.

It is true that the possibility of safe landing, through the proper execution of emergency procedures, can define the boundary between life and death of the crew involved in this critical phase of the

*Corresponding author. E-mail: fgledson@gmail.com. 
flight. For this, besides being in a safe zone of flight, the pilot needs to take a quick action, which comes from his/her experience in recognizing the failure, and their proficiency in piloting the aircraft, combined with an acceptable physical and mental load to execution the work.

Scientific researches focused on determining the workload in different environments are not recent. Although considered subjective and involving many features, several techniques to determine and delimit its variables are recurrently used in the attempt to keep in balance the relationship between the task demands and the capacities of the operator [1].

According to the International Ergonomics Association [2], "Ergonomics (or human factors) is the scientific discipline concerned with the understanding of the interactions among humans and other elements of a system, that applies theoretical principles, data and methods to design in order to optimize human well being and overall system performance".

In this sense, it is possible to consider this study inside the ergonomic scope. Mainly because it seeks to understand the work of the pilot and the load imposed to him in a flight condition that offers high risk to their physical integrity, which at the same time, their performance is vital for the success of a safe landing.

So, in the ergonomic point of view, it is expected that this work provides subsidies to further studies in this field of knowledge, as well as, to develop measures and strategies in order to increase safety in air operations with helicopters.

\section{Objective}

The objective of this paper is to present a preliminary analysis of data obtained in the initial study developed in order to validate the instruments and the research protocol that focuses on the analysis of the workload imposed on helicopter pilots during approach and landing tasks, with emergency procedures, performed in real training environment.

\section{Workload and human performance}

According to Cruz and Frutuoso [3], the term workload is a result of the need to understand that, for a given work situation, there is a permanent tension between the demands of the process and the biological and psychological capacities of workers to answer them.

Its definition is more focused on the operator than on the task, it starts manifest from the interaction between the task requirements, the circumstance under which it is performed and the skills, behaviors and perceptions of the operator [4].

Generally, workload is the result of the relationship between the demands of the task and the resource that the operator is able to spend to deal with them [5-7].

Basically, it can be classified into physical or mental. The first one refers to the set of activities of the environment and of the work activities that act on the body of the operator, as well as their respective response, such as: the physical and repetitive effort, the postures, the effects of noise, vibration, light, air quality, and so on. The second classification is characterized by mental processes necessary to perform the task, such as: perception, concentration, memory, promptitude (readiness), decision making, and so on. [8].

According to Miller and Rokicki [6], sometimes the workload exceeds the maximum capacity of the operator, and when this occurs the effectiveness of the system may be compromised. This is the importance of evaluating it.

Realizing human limitations to the demands of work, researchers have focused on developing, implementing, comparing and validating methods that could verify and measure the workload. They sought to provide a tool which was able to highlight the components of the task that determine the experience of overload, as well as measure and evaluate the intensity of different loads, thus allowing to analyze the safety and usability of a system incorporated in a workstation $(6,9-11]$.

Among the different ways of evaluating the workload, a subjective measure widely used for this purpose stands out. NASA Task Load Index Scale NASA-TLX Scale. It was developed to minimize the variability among individuals of the sample, and in order to do this, it allows the assignment of weighted to its six components, by means of paired comparisons. Then, the degree of intensity of each component is obtained, according to the perception of the respondent. Thus, it allows to know the load index of each factor, and also, through the weighted average, the overall rate of load [4]. 


\section{Methodological procedures}

\subsection{Sample}

The data presented here was obtained during the validation process of the research protocol. The group consisted of 10 volunteer pilots, with an average experience of 1123.5 flight hours (SD = 633.9), in different types of helicopters. The age ranged from 32 to 45 years (Mean $=37, \mathrm{SD}=3.6$ ), two of them were flight instructors. The group was also divided into four military pilots and six law enforcement pilots.

\subsection{Instruments}

In spite of being indicated different ways to measure the workload, and the recommendation of researchers in using more than one form of assessment in search of reference parameters and greater reliability of results [12], this preliminary study will focus on data relating to NASA TLX Scale instrument, developed by Hart and Staveland [4] and questions from the interviews after the flight.

\subsubsection{Subjective measure}

The NASA-TLX is a scale based on a multidimensional approach to assess the workload using a weighting technique to normalize each subject. It has six subscales that constitute the index, namely: mental demand, physical demand, temporal demand, performance, prolonged effort and frustration experienced [4].

For the characterization of workload in this preliminary analysis were analyzed only the subscales "physical demand" and "mental demand" of NASA-TLX Scale. The "mental demand" is understood as the level of demand that the task presented in relation to mental and perceptual activities necessary for their implementation, such as: thinking, deciding, calculating, remembering, searching, looking, and so on [8]. The "physical demand" refers to how much physical activity was required for the task suggested, regarding the need to pull, push, flip, control, hit and perform other movements with the body as a general rule [8].

\subsubsection{Descriptive analysis}

In order to understand the scores assigned in the evaluation using the NASA-TLX Scale, interviews were conducted at the end of each training session, in which the pilots answered questions about the tasks and their performance while accomplishing them.

In this study, the questions that were subject of this analysis are as follows:

"Among the tasks performed, which one you found most difficult?"

"Which one showed a higher physical demand? And why?"

"Which one showed a higher mental demand? And why?"

The reports of the pilots were transcribed according to the tasks performed. The answers were then analyzed according to the Content Analysis Technique, proposed by Bardin [8].

\subsection{Context}

Data collection occurred during the execution of emergency training that pilots must take periodically to maintain their operational skills, and was taught by the Pilot School EFAI using the helicopter model HB-350B "Squirrel".

The initial takeoff for training took place from the Morumbi helicenter, and the tasks were performed in flight above a landing area located on the banks of the Guarapiranga water reservoir in São Paulo-SP.

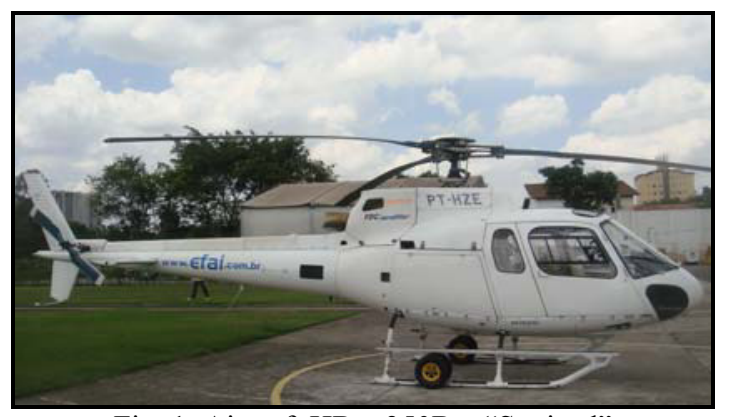

Fig. 1: Aircraft HB - 350B - "Squirrel"

(Photo registered during research)

\subsection{Data collection}

The procedures for collection of data presented in this article occurred during about 30 (thirty) hours flight performed during 3 (three) consecutive weeks.

The training was given by an experienced flight instructor for each member of the sample individually, and it lasted for about 3 hours of flight, but it was divided in two sessions, in order to avoid fatigue of the pilot.

Before the beginning of training, the pilot was instructed about the research procedures, including 
completing the NASA-TLX Scale, and answered the questionnaire for characterizing the sample.

During training, after each task chosen for the study, the instructor filled the data from NASA-TLX Scale, according to information issued by the pilot in training.

Finally, at the end of training, the pilot answered to an interview with open questions, according to predefined guidelines. This interview was recorded with a voice recorder with permission of the pilots.

\subsection{Tasks}

In this training, the instructor simulates or actually causes some kind malfunctions of any component or system, requiring the pilot's recognition of the failure and the execution of tasks to correct it. Such corrective actions are provided in the Emergency Procedures section contained in the manual of instruction for pilots of the aircraft.

During training, many of these procedures related to the malfunction were performed. For purposes of this study were selected four of them, listed below:

Task 1 (T1) - Emergency procedure for approach and landing without the operation of the hydraulic system - PSH.

Task 2 (T2) - Emergency procedure for approach and landing with tail rotor control failure - PCRC.

Task 3 (T3) - Emergency procedure for approach and landing with engine failure - PMp.

Task 4 (T4) - Emergency procedure for approach and landing with complete loss of tail rotor effectiveness - PRCp.

\section{Results}

The presentation of the results and their respective analysis will be conducted by making, whenever possible, a relationship between the results of NASATLX Scale and the answers submitted by pilots in the interviews at the end of training, thus seeking to meet the validation procedure of data.

The results of the application of NASA-TLX Scale were obtained by calculating the weighted average of the grades assigned to each of the six subscales. For purposes of this study, only the subscales "physical demand" and "mental demand" were highlighted.

In this sense, the pilots considered that the tasks T4 - PRCP (Emergency procedure for approach and landing with complete loss of tail rotor effectiveness), T2 - PCRC (Emergency procedure for approach and landing with tail rotor control failure), and T3 - PMP (Emergency procedure for approach and landing with engine failure), and have relevant mental requirement (the maximum weighted degree to be obtained is $100)$, and their mean values varied little among them $(69,1 ; 68,1 ; 67,3$, respectively). On the other hand, the T1 - PSH (Emergency procedure for approach and landing without the operation of the hydraulic system) has been identified as the one that presented a predominantly physical demand (Figure 2 ).

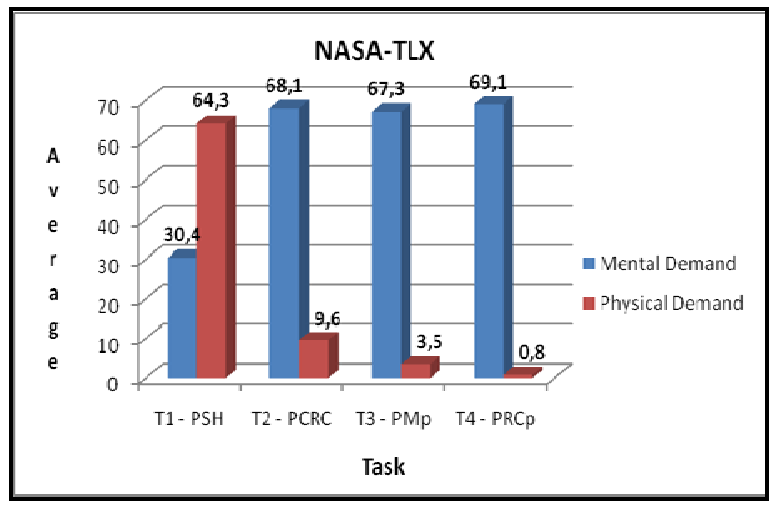

Figure 2: Distribution of the average values obtained in the evaluation of the NASA-TLX scale into subscales

"Physical Demand" and "Mental Demand"

With respect to selected questions of the interview, one of them was related to the perception that pilots had about the more difficult task. In this regard, 60\% of the group pointed out the T4 - PRCP (Emergency procedure for approach and landing with complete loss of tail rotor effectiveness) as the most difficult one (Figure 3), while T1 - PSH (Emergency procedure for approach and landing without the operation of the hydraulic system) was indicated by the group (70\%) as the least difficult one.

The T4, considered by pilots as the most demanding mental task, according to the results of NASA-TLX Scale (Figure 2), was also identified as the most difficult one. The main feature was the need for quick action by the pilot in training, in order to regain directional control of the aircraft before the height loss becomes an irreversible crash. This task was begun in the abeam of the landing site, about 300 meters away, 800 feet (ft) high from the hovering and speed around zero. In this condition the instructor pilot implemented the deficiency by pressing the left pedal in order to reach an angle where the efficiency of the tail rotor was approximately null. After that, the instructor allowed a slight increase in the speed of rotation of the fuselage of the aircraft, establishing a 
significant deficiency of directional control for the pilot in training to correct it. In addition, the trainee was forbidden to make use of the pedals, invalidating the task otherwise. After the resumption of directional control of the aircraft, the pilot in training still had to fly to the landing site and execute the autorotation landing without the aid of the pedals.

With respect to this procedure, among the pilots who considered T4 to be the most difficult task, the main factors reported as contributing most to the increase of level of difficulty was the "time pressure" and "control". For them the first factor, which was also in the reports about the task T3, was associated with the short time available for a reaction to initiate emergency procedures; and the second factor was related to the high demand for performance of the pilot to execute large amount of procedures based on physical and mental processes, in order to gain control of the aircraft. Therefore, it was evident that the time pressure and the performance required contributed to the increase of mental demands.

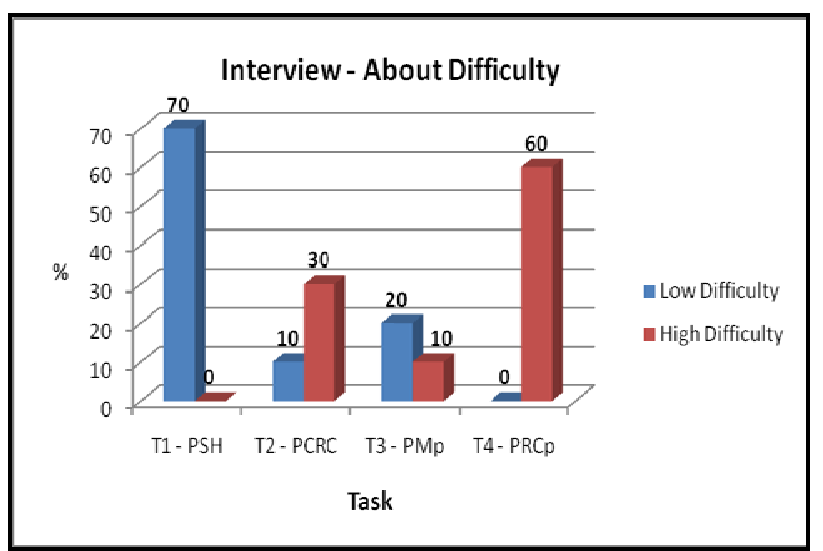

Fig. 3: Percentage distribution of answers to the question "Among the tasks performed, which one you found most difficult?"

Regarding the question about the task that had a higher physical demand, just as shown by the results obtained in the NASA-TLX Scale (Figure 2), the answers were emphatic to report that T1 - PSH (Emergency procedure for approach and landing without the operation of the hydraulic system) was predominantly a physical task and there was almost no mental demand (Figure 4).

Some of them attributed this to the slow speed in which things happened and the fact that an immediate landing was not necessary. According to them, the physical effort required due to the lack of support of the hydraulic system was prevalent at times of lower speed, and highlighted as the main physical activity the compression of the right pedal just before the landing.

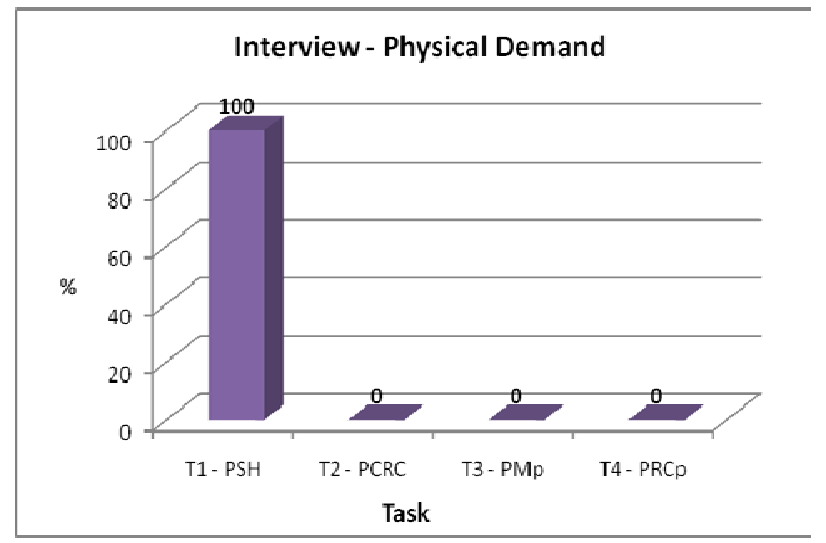

Fig. 4: Percentage distribution of answers to the question "Which one showed higher physical demands?"

As for the question asking the team to indicate the task which presented a higher mental demand, the answers were distributed between the T4 - PRCP and T2 - PCRC (40\% each) and the remaining 20\% selected to T3 - PMp (Figure 5 ). These results were also confirmed by those presented at the NASA-TLX Scale, which showed the same rating of tasks (Figure 2).

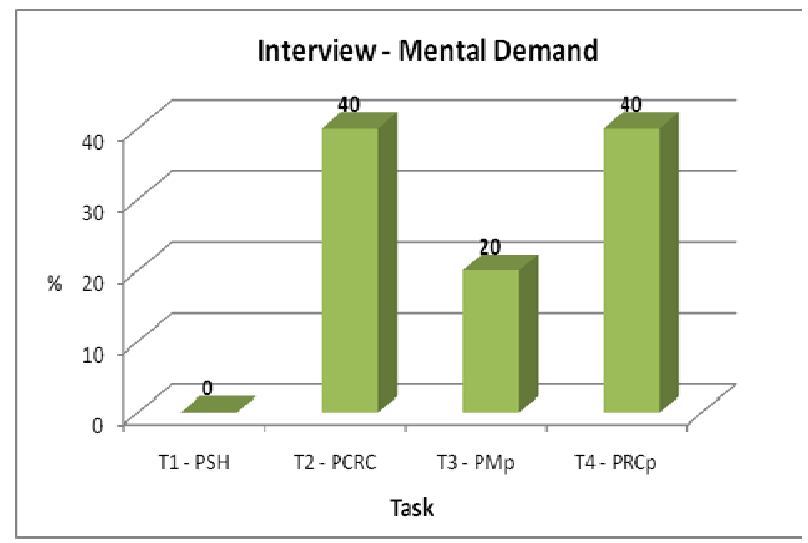

Figure 5: Percentage distribution of answers to the question "Which one showed a higher mental demand?"

In this sense, the pilots justified their answers by adding that the $\mathrm{T} 4$ demanded a great capacity of attention in order to interpret what was happening to the aircraft and decide what action to perform in a short time. The constant monitoring of the aircraft parameters and its corresponding interpretation were 
critical mental activities to the accomplishment of the task.

The T2, also ranked as the second most demanding task mental and difficulty, had the characteristic of the lack of directional control. The failure was implemented by the instructor by forbidding the pilot in training to make use of the pedals, invalidating the task otherwise. It begun in the takeoff procedure, above the landing site, at 5 feet ( $\mathrm{ft}$ ) of altitude and the velocity was close to zero. The pilot in training continued the takeoff, rising to $500 \mathrm{ft}$ and trying to maintain speed at 70 knots $(\mathrm{kt})$. After that, the pilot proceeded to a landing run without the aid of the pedals.

At that time, according to the pilots, there was a need to set up a proper landing attitude, measuring very well the application of the cyclic and collective controls to prevent tipping of the aircraft at the time of touch on the ground. This fact, according to pilots, promoted a mental demand, because it was necessary to spend a high level of attention to keep a constant monitoring of the parameters, and judge and decide the best application of commands to maintain a perfect landing attitude.

\section{Conclusions and next steps}

The concept of workload is quite broad, involving multiple variables, therefore researchers are endeavoring to restrict them in order to evaluate them in search of an easier way to determine and define, minimizing the degree of the subject's complexity.

The instruments used here constituted one of the means of assessing workload, however, if they were used in isolation, they would not have the same capacity of assessment, reducing its effectiveness.

The NASA-TLX Scale proved to be valid as an instrument of measurement, in the way that the results obtained, in assessing the mental and physical demands, are compatible with the view of pilots when asked about the predominant type of demand on each task performed.

Since the objective of this paper was guided much more in analyzing than dimensioning the demands of the pilot in an emergency phase of flight, the reports provided by them at the end of the tasks were of fundamental importance for a better understanding of the work of the pilot, in the way that it provided further explanation of the difficulties encountered and the strategies used to solve the problems.
This detailed description exposed by the pilots in their workplace, the processes by which he understood that the new situation brought in the aircraft, as well as the way they interacted with it, can provide valuable information, not only regarding workload but also in order to contribute to increased levels of flight safety.

Preliminarily, through these descriptions, it was possible to identify the "time pressure" as a relevant factor in contributing to increase mental demand in emergency situations in flight, and that, this increase is reflected in the request of mental processes such as: identification of failures, attention and monitoring parameters.

Although this study was developed in real flight training, it took place in a controlled environment. Military air operations and law enforcement operations in helicopters are examples of activities that require high performance of the operator. This is due to the fact that the activity occurs in an operating scenario with high potential of spontaneous or induced hostility, high interaction with occurrences on the ground, many resources to be managed, aside from the common requirements of the air activity. It becomes clear that these ergonomic approaches in the workplace are desirable, and would contribute not only to improve the workplace, but also to increase flight safety.

This article consisted of the presentation of data obtained during the preliminary survey carried out in order to outline the methodological procedures to be implemented during the data collection study about the workload in helicopter pilots during emergency situations. The expansion of the sample and the inclusion of other physiological tools, such as the measure of heart rate and heart rate variability, will make possible the production of knowledge about the effects of these types of emergencies on pilot performance and, if possible, the identification of strategies that can minimize the negative effects that, by chance, affect flight safety.

\section{Acknowledgement}

This study was developed thanks to the voluntary participation of military pilots from Brazilian armed forces (navy, air force and army) and the law enforcement. A special acknowledgement to the pilot instructor and the commander of the EFAI School, a Latin America center of excellence in helicopter training and education of pilots, without which this 
work could not have been accomplished. This study was supported by Brazilian National Secretary of Public Security, motivated by the laudable idea of promoting knowledge to enhance their efficiency.

\section{References}

[1] S.L.O. Ribeiro and M.C.R. Vidal, Cockpit work analysis according to pilot subjective perception, in: 16th World Congress on Ergonomics. Proceedings of IEA2006 Congress. Elsevier Ltd., Maastricht, Holland, 2006.

[2] International Ergonomics Association (IEA), Ergonomics definition, Available at http://www.iea.cc/01 what/ What $\% 20$ is\%20Ergonomics.html, accessed March $15^{\text {th }} \overline{2011 .}$

[3] R.M Cruz and T.J. Frutuoso, Mensuração da carga de trabalho e sua relação com a saúde do trabalhador [Measurement of the workload and its relationship with the worker's health], in. Rev. Bras. Med. Trab., Belo Horizonte, Minas Gerais, 3(1), (2005), pp. 29-36

[4] S.G Hart and L.E. Staveland, Development of NASA-TLX (Task Load Index): Results of empirical and theoretical research, in: P.A Hancock and N. Meshkati (Eds) Human mental workload. Elsevier, Amsterdam, Holland, 1988, pp. 139-184.

[5] D. De Waard, The measurement of drivers' mental workload. Haren, The Netherlands, Ph.D. Dissertation, University of Groningen, Trafic Research Centre, 1996.
[6] J.C. Miller and S.M. Rokicki, Psychophysiological test methods and procedures, in: T. G. O'Brien and S.G. Charlton, (Eds) Handbook of human factors testing and evaluation. LEA, New Jersey, 1996, pp. 135-155.

[7] M.C.R. Vidal, A ergonomia na empresa: útil, prática e aplicada, [The ergonomics at company: useful, practical and applied], Editora Virtual Cientifica, Rio de Janeiro, 2001.

[8] S.L.O. Ribeiro, Carga de trabalho e automação: estudo da cabine de voo do ponto de vista da ergonomia cognitiva, [Workload and automation: a cognitive ergonomic study concerning aircraft cockpit design], Ph.D. Dissertation, Universidade Federal do Rio de Janeiro, 2003.

[9] W.H. Corwin, D.L. Sandry-Garzy, M.H Biferno et al. Assessment of crew and measurement methods, and results. (WRDC-TR-89-7006), Wright Research and Development Center, Ohio, 1989.

[10] P. Park, and D. Cha, Comparison of subjective mental workload assessment techniques for the evaluation of invehicle navigation system usability, in: Proceedings of Session T56 of the 5th World Congress on Intelligent Transport Systems, Seoul, 1998.

[11]R.W. Schvaneveldt, G.B Reid, R.L Gomez and S. Rice, Modeling mental workload, in: Cognitive Technology, 3 (1998), 9-31.

[12]F.P. Corrêa, Carga mental e ergonomia, [Mental workload and ergonomics], M.Sc Dissertation. Engenharia de Produção, Universidade Federal de Santa Catarina, UFSC, Florianópolis, 2003. 